\title{
A light leptophobic Z' in polarized hadronic collisions
}

\author{
P. Taxil and J.-M. Virey \\ Centre de Physique Théorique*, C.N.R.S. - Luminy, Case 907 \\ F-13288 Marseille Cedex 9, France \\ and \\ Université de Provence, Marseille, France
}

\begin{abstract}
Theoretical and phenomenological arguments are in favor of an elusive new neutral vector boson $Z^{\prime}$ with a relatively low mass, chiral couplings to ordinary quarks and whose couplings to ordinary leptons are suppressed (leptophobia). We point out that this new particle could induce some parity violating spin asymmetries which could be measured soon at the Brookhaven Relativistic Heavy Ion Collider (RHIC), running part of the time as a polarized hadronic collider.
\end{abstract}

PACS Numbers : 12.60.Cn; 13.87.-a; 13.88.+e; 14.70.Pw

Key-Words : New Gauge bosons, Jets, Polarization.

Number of figures : 3

July 1998

CPT-98/P.3667

anonymous ftp or gopher : cpt.univ-mrs.fr

*Unité Propre de Recherche 7061

E-mail : Taxil@cpt.univ-mrs.fr ; Virey@cpt.univ-mrs.fr 


\section{Introduction}

One of the simplest extensions of the Standard Model (SM) is the addition of an extra $U(1)^{\prime}$ gauge factor to the $S U(3) \times S U(2) \times U(1)$ structure. If the symmetry breaking occurs at a scale not far from the electroweak scale, this leads to the existence of a new neutral gauge boson $Z^{\prime}$ at a mass accessible to forthcoming experiments.

In recent years, some discrepancies with respect with the Standard Model expectations observed at LEP and/or FNAL have triggered a lot of studies involving a possible $Z^{\prime}$ whose couplings to ordinary leptons could be very small (leptophobia).

Although the discrepancy in the $Z \rightarrow b \bar{b}$ sector has not completely disappeared, the improved agreement between the latest data [1] and the SM expectations has weakened the phenomenological motivations for that object (see e.g. [2]). However, the interest on such a leptophobic $Z^{\prime}$ goes far beyond the tentative for explaining the LEP and CDF data. Various models, based on Grand Unification and/or Superstring theory, display a parameter space which allows or favors a new neutral gauge boson with very small couplings to leptons. On the other hand, leptophobia still represents an attractive possibility allowing the existence of new physics at an accessible energy scale without any contradiction with the present data.

It has been emphasized in refs. [3, 4, 5, 6] that some non minimal SUSY models with an additional $U(1)^{\prime}$ imply the presence of a relatively light $Z^{\prime}: M_{Z^{\prime}}<1 \mathrm{TeV} / c^{2}$. Indeed, a class of models, driven by a large trilinear soft SUSY-breaking term, prefers the range $M_{Z} \leq M_{Z^{\prime}} \leq 400 \mathrm{GeV} / c^{2}$ along with a very small mixing with the standard $Z^{\circ}$. This opens some very interesting possibilities for phenomenology provided that this new vector boson displays leptophobic couplings to remain compatible with present data.

A leptophobic $Z^{\prime}$ is particularly elusive as far as conventional direct searches via DrellYan pair production at a $p \bar{p}$ collider are concerned. It is of some importance to consider other possible manifestations of this new boson.

If produced in hadronic collisions, the new $Z^{\prime}$ can decay into exotic fermions whose presence is necessary for anomaly cancellations [7]. Such decays can yield some anomalous events which are, however, difficult to interpret.

An other interesting feature has been pointed out by Rosner [7] : the direct $Z^{\prime}$ couplings to quarks (which can be generation dependent or not) often break chiral symmetry at a variance with QCD which is left-right symmetric. This can yield a substantial forwardbackward asymmetry $A_{F B}$ in $p \bar{p} \rightarrow Z^{\prime} \rightarrow f \bar{f}$ events. Such measurements are particularly difficult in the absence of outgoing leptons (leptophobia) since it is mandatory to measure the charge of the outgoing particle or jet. Moreover, in some case like in the model described in [7], $A_{F B}=0$ in the production of down(bottom)-type quarks which forbids the opportunity of using $b$-tagging.

There is an other way to be sensitive to chiral couplings, namely the measurement of a parity-violating (PV) spin effect in polarized hard collisions of hadrons, in particular in the production of jets [8]. In fact, within two years, the RHIC Spin Collaboration (RSC) [9] will start running the Relativistic Heavy Ion Collider at Brookhaven National 
Laboratory part of the time in the $\vec{p} \vec{p}$ mode, with a high (70\%) degree of polarization and with a very high luminosity $\mathcal{L}=2.10^{32} \mathrm{~cm}^{-2} \mathrm{~s}^{-1}$. The physics goals of the collaboration have been discussed in a recent workshop [10].

As soon as one can find some arguments for -i) an extra $U(1)^{\prime}$ gauge factor leading to a relatively light $Z^{\prime}$, -ii) a parameter space which favors some very weak couplings of this $Z^{\prime}$ to conventional leptons, -iii) a model in which an asymmetry in the left and right-handed couplings to light quarks is preferred or at least allowed, then it is extremely interesting to explore the consequences on the spin observables which will be measured soon, with a high degree of precision, by the RHIC Spin Collaboration.

In the following section we will summarize the theoretical motivations for a light and leptophobic $Z^{\prime}$ with chiral couplings to quarks. We also give the parameter space which follows from the models we consider, along with the present experimental direct limits on the mass of the $Z^{\prime}$ and the strength of its coupling to light quarks. The effects of the new amplitudes on the spin asymmetries in inclusive jet production at the polarized RHIC collider are presented in Section 3, along with the bounds on the parameter space which could be obtained. We give our conclusions in Section 4.

\section{Theoretical motivations and the parameter space}

We will consider a new neutral vector boson $Z^{\prime}$ which couples to $u$ - and $d$ - type quarks with the following structure :

$$
\mathcal{L}_{Z^{\prime}}=\kappa \frac{g}{2 \cos \theta_{W}} Z^{\prime \mu} \bar{q} \gamma_{\mu}\left[C_{L}^{q}\left(1-\gamma_{5}\right)+C_{R}^{q}\left(1+\gamma_{5}\right)\right] q
$$

for each given quark flavor $q$, the parameter $\kappa=g_{Z^{\prime}} / g_{Z}$ being of order one.

We will not discuss any more the phenomenological models with strong couplings and $M_{Z^{\prime}}=1 \mathrm{TeV} / c^{2}$ which were built [11] to explain the old LEP (+CDF) data. Spin asymmetries at RHIC for these particular models have been already discussed in Ref. 12 .

A theoretically motivated leptophobic $Z^{\prime}$ with chiral couplings is present in many string inspired models : in particular we will consider a version due to Lopez and Nanopoulos [13] (model A) of the flipped-SU(5) model [14] and a generalized $E_{6}$ model with kinetic-mixing |15] (model B).

- In model $(\mathrm{A})$, the particle content of the standard model is contained in the representations : $F=\left(\mathbf{1 0}, \frac{1}{2}\right)=\left\{Q, d^{c}, \nu^{c}\right\}, \bar{f}=\left(\overline{\mathbf{5}},-\frac{3}{2}\right)=\left\{L, u^{c}\right\}, l^{c}=\left(\overline{\mathbf{1}}, \frac{5}{2}\right)=\left\{e^{c}\right\}$.

Leptophobia is achieved when $\bar{f}$ and $l^{c}$, which contain the standard model leptons, are uncharged under the new $U(1)^{\prime}$. As a consequence (see [13]) the quark couplings verify $C_{L}^{u}=C_{L}^{d}=-C_{R}^{d}=1 /(2 \sqrt{3})$ and $C_{R}^{u}=0$. Therefore parity is maximally violated in the up-quark sector and is conserved in the down-quark sector since the resulting coupling is purely axial. 
- Model (B) goes beyond the traditional parametrization in terms of the $U(1)$ 's combination in $E_{6}$ models (for a review of $E_{6}$ models see [16]). In fact, it was noticed some years ago [17] that it was possible within $E_{6}$ models, to find a particular combination of charges leading to a suppression of the $Z^{\prime}$ couplings to ordinary leptons (see [7] for a recent discussion). In Ref.[15] one considers that kinetic mixing (KM) between the field strength of weak hypercharge $B_{\mu \nu}$ and the field strength of the new $U(1)^{\prime}$ occurs. In the interesting case of KM between the so-called $U(1)_{\eta}$ and $U(1)_{Y}$, one gets leptophobia when, for each fermion $f$, the $Z^{\prime}$ couples to a combination $Q^{\prime f}=Q_{\eta}^{f}+\delta Y_{W}$ with $\delta=-1 / 3$. This yields the quark couplings : $C_{L}^{u}=C_{L}^{d}=C_{R}^{d}=-\frac{1}{2} C_{R}^{u}=-\frac{5}{18} \sin \theta_{W}$.

Note that to maintain $\kappa$ of order one in eq.(1), the usual GUT factor $\sqrt{\frac{5}{3}} \sin \theta_{W}$ has been included in the $C_{L, R}^{q}$ 's couplings.

Since a leptophobic $Z^{\prime}$ appears in several other string derived models [5, 18] it is valuable to consider a more general situation where the $Z^{\prime}$ couplings to ordinary quarks are less constrained. We will nevertheless assume $S U(2)_{L}$ invariance which imposes $C_{L}^{u}=$ $C_{L}^{d} \equiv C_{L}$. Then, we are left with three parameters : $C_{L}, C_{R}^{u}$ and $C_{R}^{d}$. Of course in some models the couplings are generation dependent : since we will be interested in ordinary jets, this point is not relevant for our analysis.

Non-SUSY leptophobic models have also been constructed [19, 20]. Again there is a large freedom in the precise values for the $Z^{\prime}$ couplings to light quarks. We will consider for illustration the special case introduced in Ref. [20] where the $Z^{\prime}$ is purely right-handed (model C) : $C_{L}=0, C_{R}^{u}=C_{R}^{d}=1 / 3$ (to be consistent with our notations we have divided the value given in 20 by a factor 3 to keep the corresponding $\kappa \approx 1$ ).

The constraints on models (A) and (B) which are coming from updated electroweak data have been studied very recently [21]. It turns out that for a small value of the $Z^{\circ}$ $Z^{\prime}$ mixing angle $\xi$ the $Z^{\prime}$ mass remains essentially an unconstrained parameter in case of pure leptophobia. From the theoretical side a very small mixing angle is natural in the scenario advocated in [4]. One gets the same behaviour when the new $U(1)^{\prime}$ does not mix with the SM gauge group like within a class of models which can include model(A) [13]. As a consequence we have neglected the $Z^{\circ}-Z^{\prime}$ mixing angle $\xi$ which is in principle present in all the $C_{L, R}^{q}$ couplings and whose effect on the calculations described below would be very tiny.

Direct constraints on $M_{Z^{\prime}}$ are coming from $p-\bar{p}$ collider experiments analyzing the dijet cross section: UA2 [22], CDF [23] and D0 [24]. These limits are usually displayed in term of the so-called "sequential standard model $Z^{\prime}$ " with $\kappa=1$. It is not difficult to perform an extrapolation for a reasonable range of $\kappa$ values and for a larger class of $Z^{\prime}$ models with various $C_{L, R}^{q}$ couplings. We have displayed the limits in Fig.1 for models (A) and (B) assuming a $100 \%$ branching ratio of the $Z^{\prime}$ into ordinary jets. One can notice that the $Z^{\prime}$ mass is not constrained in these models as soon as $\kappa<0.95$. Also, and this is true in any leptophobic model, some windows are present around $M_{Z^{\prime}}=300 \mathrm{GeV} / c^{2}$ and below $M_{Z^{\prime}}=100 \mathrm{GeV} / c^{2}$.

Therefore, one can see that present data are not excluding a $Z^{\prime}$ as light as the one which is advocated in recent papers based on weak-scale supersymmetry [3, 4, 5, 6]. As discussed 
by Lykken [5] this scenario could take place for model (A). This is also the case for model (B), according to Refs. [6], if additional matter from an extra $\mathbf{7 8}$ representation of $E_{6}$ is involved. Finally, we will also consider the very special case where the $Z^{\prime}$ is degenerate in mass with the SM $Z^{\circ}$. This can be accepted in the framework of the trilinear scenario of Ref. [1] and it was stressed in Ref. 25] that such a leptophobic boson could explain the apparent discrepancy between the LEP and SLAC values of $\sin ^{2} \theta_{W}$. To summarize, we will focus on a mass range $M_{Z} \leq M_{Z^{\prime}} \leq 400 \mathrm{GeV}$ which could give spectacular effects in PV spin asymmetries at RHIC.

\section{Calculation and results}

At RHIC, running in the $\vec{p} \vec{p}$ mode, it will be possible to measure with a great precision the double helicity PV asymmetry :

$$
A_{L L}^{P V}=\frac{d \sigma_{(-)(-)}-d \sigma_{(+)(+)}}{d \sigma_{(-)(-)}+d \sigma_{(+)(+)}}
$$

where the signs \pm refer to the helicities of the colliding protons. The cross section $d \sigma_{\left(\lambda_{1}\right)\left(\lambda_{2}\right)}$ means the one-jet production cross section in a given helicity configuration, $p_{1}^{\left(\lambda_{1}\right)} p_{2}^{\left(\lambda_{2}\right)} \rightarrow$ jet $+X$, estimated at $\sqrt{s}=500 \mathrm{GeV}$ for a given jet transverse energy $E_{T}$, integrated over a pseudorapidity interval $\Delta \eta=1$ centered at $\eta=0$.

The $Z^{\prime}$ will generate new amplitudes in quark-quark scattering which is the dominant subprocess in the $E_{T}$ range we consider. At LO these amplitudes will interfere with the one-gluon exchange amplitude and also with the amplitudes due to SM gauge boson exchanges. This was described already in Ref. [12] and all the LO cross sections for the subprocesses can be found in Ref. 26].

In fact, $95 \%$ of the PV effect due to the new boson will come from $Z^{\prime}$ - gluon interference terms involving the scattering of $u$ quarks in the $t$-channel.

In short notations :

$$
A_{L L}^{P V} \cdot d \sigma \simeq F \int\left(\left(C_{L}^{u}\right)^{2}-\left(C_{R}^{u}\right)^{2}\right)\left[u\left(x_{1}, \mu^{2}\right) \Delta u\left(x_{2}, \mu^{2}\right)+\Delta u\left(x_{1}, \mu^{2}\right) u\left(x_{2}, \mu^{2}\right)\right]
$$

where $F$ is a positive factor given by

$$
F=\frac{32}{9} \alpha_{s} \alpha_{Z} \hat{s}^{2} \operatorname{Re}\left(\frac{1}{\hat{t} D_{Z^{\prime}}^{\hat{u}}}+\frac{1}{\hat{u} D_{Z^{\prime}}^{\hat{t}}}\right)
$$

where $\alpha_{Z}=\alpha / \sin ^{2} \theta_{W} \cos ^{2} \theta_{W}$ and

$$
D_{Z^{\prime}}^{\hat{t}(\hat{u})}=\left(\hat{t}(\hat{u})-M_{Z^{\prime}}^{2}\right)+i M_{Z^{\prime}} \Gamma_{Z^{\prime}}
$$

In eq.(3) $\Delta u\left(x, \mu^{2}\right)=u^{+}\left(x, \mu^{2}\right)-u^{-}\left(x, \mu^{2}\right)$ where $u^{ \pm}\left(x, \mu^{2}\right)$ are the distributions of the polarized $u$ quarks, either with helicity parallel $(+)$ or antiparallel (-) to the parent proton 
helicity. Summing $u^{+}$and $u^{-}$one recovers the unpolarized distribution $u\left(x, \mu^{2}\right)$. Concerning these spin-dependent distributions (evaluated at $\mu=E_{T}$ ), we have used the ones of GRSV [27]. Note that the first part of the polarized RHIC Spin program itself [9] will greatly improve our knowledge of all the distributions $\Delta q_{i}$ 's and $\Delta \bar{q}_{i}$ 's (see Ref. [28] for a recent analysis). In the present analysis, the $Z^{\prime}$ couplings to quarks remain in a "weak" range (of the same order as the SM $Z^{\circ}$ couplings) : this is at a variance with the phenomenological attempts of Refs. [11]. As a consequence the $Z^{\prime}$ width $\Gamma_{Z^{\prime}}$ remains in the range of $1-10 \mathrm{GeV} / c^{2}$ (neglecting possible light exotica), the precise value having no influence on our results.

We present in Fig.2 the result of our calculation (taking all the terms, dominant or not, into account) for $A_{L L}^{P V}$ versus $E_{T}$ in $\overrightarrow{p p}$ collisions at RHIC. For illustration we compare the SM asymmetry, which is due to small QCD-electroweak interference terms [29], to the non-standard one with a $Z^{\prime}$ of mass 90 or $200 \mathrm{GeV} / c^{2}$ for models (A) and (B) and 300 $\mathrm{GeV} / c^{2}$ for model $(\mathrm{C})$. The bump in the standard $A_{L L}^{P V}$ at $E_{T} \approx M_{Z, W} / 2$ corresponds to the vicinity of the corresponding "jacobian peak" in the production cross section. We observe the same behaviour at $E_{T} \approx 100 \mathrm{GeV}$ for $M_{Z^{\prime}}=200 \mathrm{GeV} / c^{2}$. Practically, it will be difficult to explore the $E_{T}$ region below $45 \mathrm{GeV}$ with the RHIC detectors due to experimental cuts [30]. However, above $E_{T}=50 \mathrm{GeV}$, on can see that a high precision measurement can be performed. The error bars correspond to the statistical error with an integrated luminosity $\mathcal{L}_{1}=800 \mathrm{pb}^{-1}$ which can be achieved at RHIC in a few months running.

Clearly, if a deviation from the SM is seen in $A_{L L}^{P V}$, the sign of this deviation can allow to separate easily a model dominated by left-handed couplings to $u$ quarks (like model (A)) from a model dominated by right-handed couplings like model (B) or (C). Of course the results are impressive because the mass of the leptophobic $Z^{\prime}$ is light. On the other hand, we have taken a value of $\kappa$ around 1.5, when it is allowed by present data, or around 1 if not. Both values are compatible with "weak" $Z^{\prime}$ couplings to quarks (to be compared with the models in [11, 12]).

We have shown in Fig.1 the limits on the parameter space $\left(\kappa, M_{Z^{\prime}}\right)$ one can obtain from $A_{L L}^{P V}$ with the integrated luminosities $\mathcal{L}_{1}=800 p b^{-1}$ and $\mathcal{L}_{2}=3200 p b^{-1}$ for models (A) and (B). One can see that the hole centered on $M_{Z^{\prime}} \approx 300 \mathrm{GeV} / c^{2}$ is now fully covered, along with the low mass or "degenerate" case $M_{Z^{\prime}} \approx M_{Z}$. Fig.3 is more general since it is model independent. It represents some exclusion contours at $95 \%$ C.L. in the plane $\left(\kappa . C_{L}, \kappa . C_{R}^{u}\right)$ for two values of the $Z^{\prime}$ mass. The circles represent some estimates of the present experimental constraints on this parameter space assuming some simple additional relations $\left(\left(C_{R}^{d}\right)^{2}=\left(C_{R}^{u}\right)^{2}\right)$ between the couplings entering the dijet cross section. This assumption is not necessary to get the contours obtained from $A_{L L}^{P V}$ since the $d$-quark couplings have a very small influence on this asymmetry.

The particular models (A), (B) and (C) are represented by some particular points on the same plot for $\kappa=1$. Moving away from this $\kappa$ value results in a translation along a straight line as shown. One can see that the measurement of $A_{L L}^{P V}$ at RHIC will strongly reduce the allowed parameter space, in particular in the region around $M_{Z^{\prime}}=300 \mathrm{GeV} / c^{2}$ 
which is poorly constrained up to now. Only when parity is restored, that is along the diagonals, this kind of measurement cannot provide us some useful information.

\section{Conclusions}

Interest is growing on the potentialities offered by the polarized RHIC collider to get a handle on new phenomena thanks to precision measurements of spin asymmetries [10]. We had first investigated in Ref. [12 the influence on $A_{L L}^{P V}$ in $\vec{p} \vec{p}$ collisions of a purely phenomenological $Z^{\prime}$ with strong couplings to quarks and a mass around $1 \mathrm{TeV} / c^{2}$. Now it turns out that various theoretical models are in favor of a lighter and leptophobic new neutral gauge boson displaying chiral couplings to quarks. We have checked in this paper that it is particularly relevant to search for some effects in the experimental conditions of RHIC. In particular, the quite poor information available from the $p \bar{p}$ dijet experiments could be complemented for a wide part of the parameter space. Moreover, in case of a positive signal it is possible to get immediately an information on the chiral structure of the new interaction. However it is still not possible to discriminate between a SUSY or a non-SUSY origin of the new $Z^{\prime}$.

Finally, it is interesting to note that a general prediction of all the leptophobic models we have investigated (which assume a trilinear Yukawa term for $u$-type quarks) is that $C_{L}^{u} \neq C_{R}^{u}$ since the Higgs doublet $H_{U}$ is assumed to be charged under the group $U(1)^{\prime}$. This implies that parity is violated in the $u$-quark sector except for the very special axial case : $C_{L}^{u}=-C_{R}^{u}$. On the other hand, parity is conserved for $d$-quark couplings in the minimal two- Higgs doublets models [15], which is not a priori the case for non-minimal models (see e.g. Refs. 4 , 20]). Unfortunately, as we have said before, $d$-quark interactions are essentially masked in $\overrightarrow{p p}$ collisions. It will be mandatory to perform polarized neutronneutron collisions to get a complementary information : at RHIC this could be realized with polarized ${ }^{3} \mathrm{He}$ beams as discussed recently [31]. Motivated by these arguments, and also by our earlier work on the charged gauge boson sector [32], we are strongly in favor of a complete polarization program at RHIC.

\section{Acknowledgments}

J.M.V. acknowledges the warm hospitality at the RIKEN-BNL Research center where part of this work has been performed. Thanks are due to G. Bunce, G. Eppley, B. Kamal, N. Saito, M. Tannenbaum and W. Vogelsang for fruitful discussions. P.T. wishes to thank Yves Bigot for kind referencing. 


\section{References}

[1] The LEP Collaborations Aleph, Delphi, L3, Opal, the LEP Electroweak Working Group and the SLD Heavy Flavor Group, CERN-PPE/97-154.

[2] M.L. Mangano, proceedings of the Warsaw Conference ICHEP96, p. 1332.

[3] M. Cvetič and P. Langacker, Phys. Rev. 54 (1996) 3570.

[4] M. Cvetič, D.A. Demir, J.R. Espinosa, L. Everett and P. Langacker, Phys. Rev. 56 (1997) 2861.

[5] J. D. Lykken in Snowmass 1996, ed. D.G. Cassel, L. Trindle Gennari and R.H. Siemann, p. 891.

[6] P. Langacker and J. Wang, hep-ph/9804438, see also P. Langacker hep-ph/9805486.

[7] J.L. Rosner, Phys. Lett. B387 (1996) 113.

[8] M. Tannenbaum, in Polarized Collider Workshop, J. Collins, S.F. Heppelmann and R.W. Robinett eds, AIP Conf. Proceedings 223, AIP, New York, 1990, p. 201.

[9] G. Bunce et al. (RHIC Spin Collaboration), Polarized protons at RHIC, Particle World, 3 (1992) 1 ; RSC, Letter of intent, April 1991 and RSC (STAR/PHENIX) letter of intent update, August 1992, BNL Reports, unpublished.

[10] Proceedings of the RIKEN-BNL research center workshop, april 1998, BNL Report 65615.

[11] G. Altarelli et al., Phys. Lett. B375 (1996) 292 ; P. Chiappetta et al., Phys. Rev. D54 (1996) 789.

[12] P. Taxil and J.-M. Virey, Phys. Lett. B383, 355 (1996).

[13] J.L. Lopez and D.V. Nanopoulos, Phys. Rev. D55 (1997) 397.

[14] I. Antoniadis, J. Ellis, J.S. Hagelin and D.V. Nanopoulos, Phys. Lett. B194 (1987) 321.

[15] K.S. Babu, C. Kolda and J. March-Russell, Phys. Rev. D54 (1996) 4635 ; D57 (1998) 6788.

[16] J.L. Hewett and T.G. Rizzo, Phys. Reports, 183, 193 (1989).

[17] F. del Aguila, G. Blair, M. Daniel and G.G. Ross, Nucl. Phys. B283 (1987) 50.

[18] A.E. Faraggi and M. Masip., Phys. Lett. B388 (1996) 524.

[19] K. Agashe, M. Graesser, I. Hinchliffe and M. Suzuki, Phys. Lett. B385 (1996) 218. 
[20] H. Georgi and S.L. Glashow, Phys. Lett. B387, 341 (1996).

[21] Y. Umeda, G.C. Cho and K. Hagiwara, hep-ph/9805447, hep-ph/9805448.

[22] J. Alitti et al., Zeit. f. Phys. C49 (1991), 17 ; Nucl. Phys. B400 (1993), 3.

[23] F. Abe et al., Phys. Rev. D55 (1997) R5263.

[24] B. Abbott et al., FERMILAB-Conf-97/356-E.

[25] F. Caravaglios and G.G.Ross, Phys. Lett. B346 (1995), 159.

[26] C. Bourrely, J. Ph. Guillet and J. Soffer, Nucl. Phys. B361 (1991) 72.

[27] M. Glück, E. Reya, M. Stratman and W. Vogelsang, Phys. Rev. D53 (1996), 4775.

[28] J. Soffer and J.-M. Virey, Nucl. Phys. B509 (1998) 297.

[29] M. Abud, R. Gatto and C.A. Savoy, Ann. Phys. (NY) 122 (1979) 219 ; U. Baur, E.W.N. Glover and A.D. Martin, Phys. Lett. B232 (1989) 519.

[30] G. Eppley in Ref.[10], p. 301.

[31] E. Courant in Ref.[10], p. 275.

[32] P. Taxil and J.-M. Virey, Phys. Lett. B404, 302 (1997); J.-M. Virey in Ref.[10], p. 293. 


\section{Figure captions}

Fig. 1 Bounds on the parameter space $\left(\kappa, M_{Z^{\prime}}\right)$ in models $\mathrm{A}$ and B. Contours are from the dijet cross section analysis in $p \bar{p}$ collisions at CERN (UA2) (90\% C.L.) and FNAL (CDF,D0) (95\% C.L.). The dotted (dashed) line corresponds to the predicted limit at (95\% C.L.) from $A_{L L}^{P V}$ in polarized $p p$ collisions at RHIC with a c.m. energy of $500 \mathrm{GeV}$ and an integrated luminosity $\mathcal{L}_{1}=800 p b^{-1}\left(\mathcal{L}_{2}=3200 p b^{-1}\right)$.

Fig. $2 A_{L L}^{P V}$ for one-jet inclusive production, versus $E_{T}$, for polarized $p p$ collisions at RHIC. The plain curve is the SM expectation, the dotted curves correspond to the "degenerate" case $M_{Z^{\prime}}=M_{Z}$ in model (A) (upper curve) and model (B) (lower curve). Same for the dashed curves with $M_{Z^{\prime}}=200 \mathrm{GeV} / c^{2}$. The dash-dotted curve corresponds to model (C) with $M_{Z^{\prime}}=300 \mathrm{GeV} / c^{2}$. The error bars correspond to the statistical error with the luminosity $\mathcal{L}_{1}$.

Fig. 3 Contour plots at 95\%C.L. in the plane $\left(\kappa . C_{L}, \kappa . C_{R}^{u}\right)$ from $A_{L L}^{P V}$ measured with the integrated luminosity $\mathcal{L}_{2}$. Plain (dashed) curves are for $M_{Z^{\prime}}=200(300) \mathrm{GeV} / c^{2}$. The circles correspond to exclusion limits from present collider data. The black triangle, square and disk correspond to models (A), (B) and (C) respectively for $\kappa=1$. 


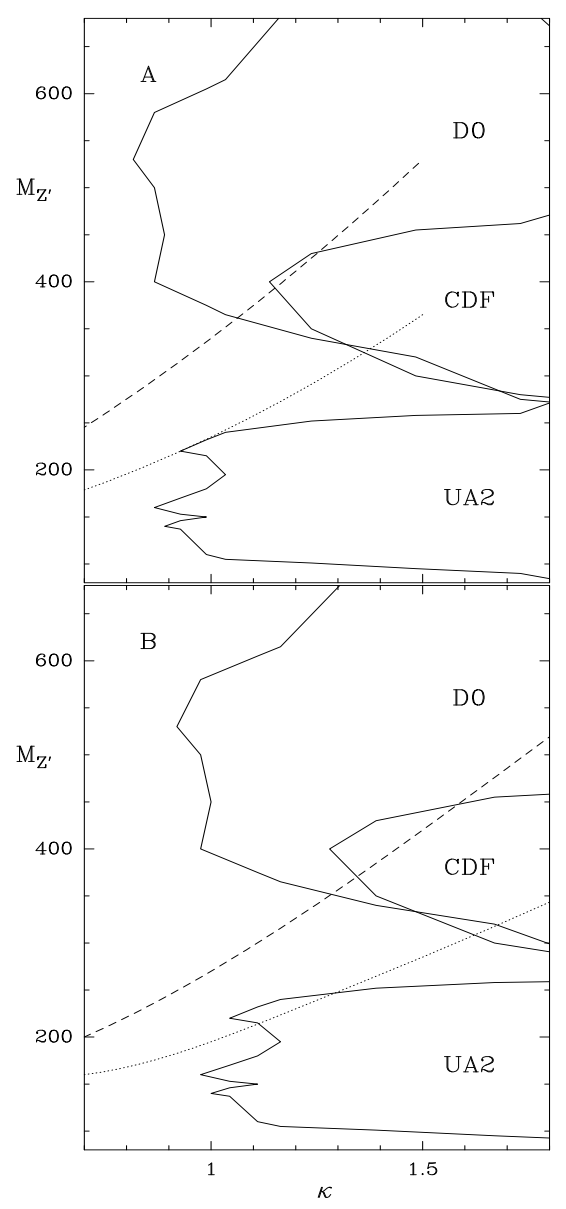

Fig 1 


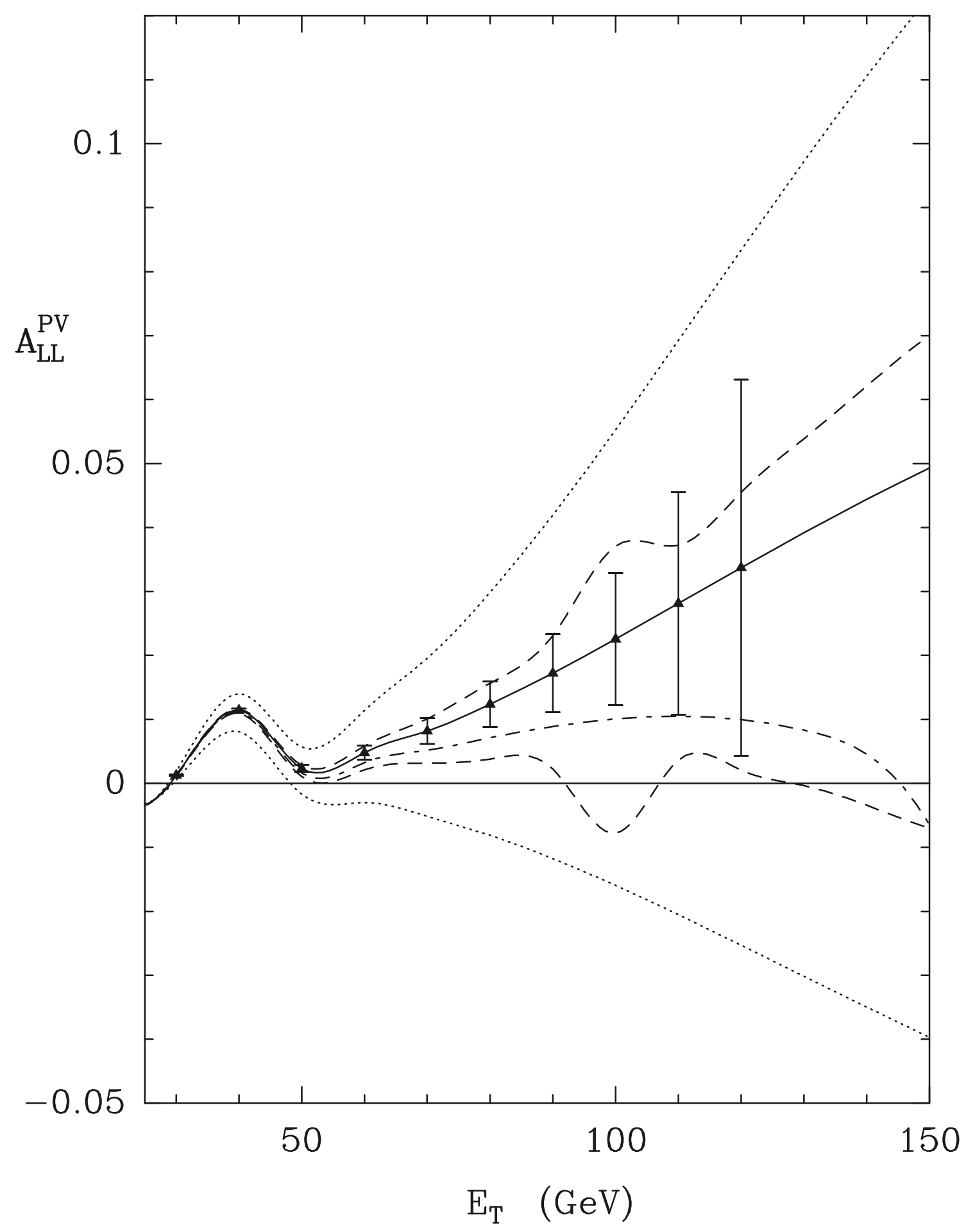

Fig 2 


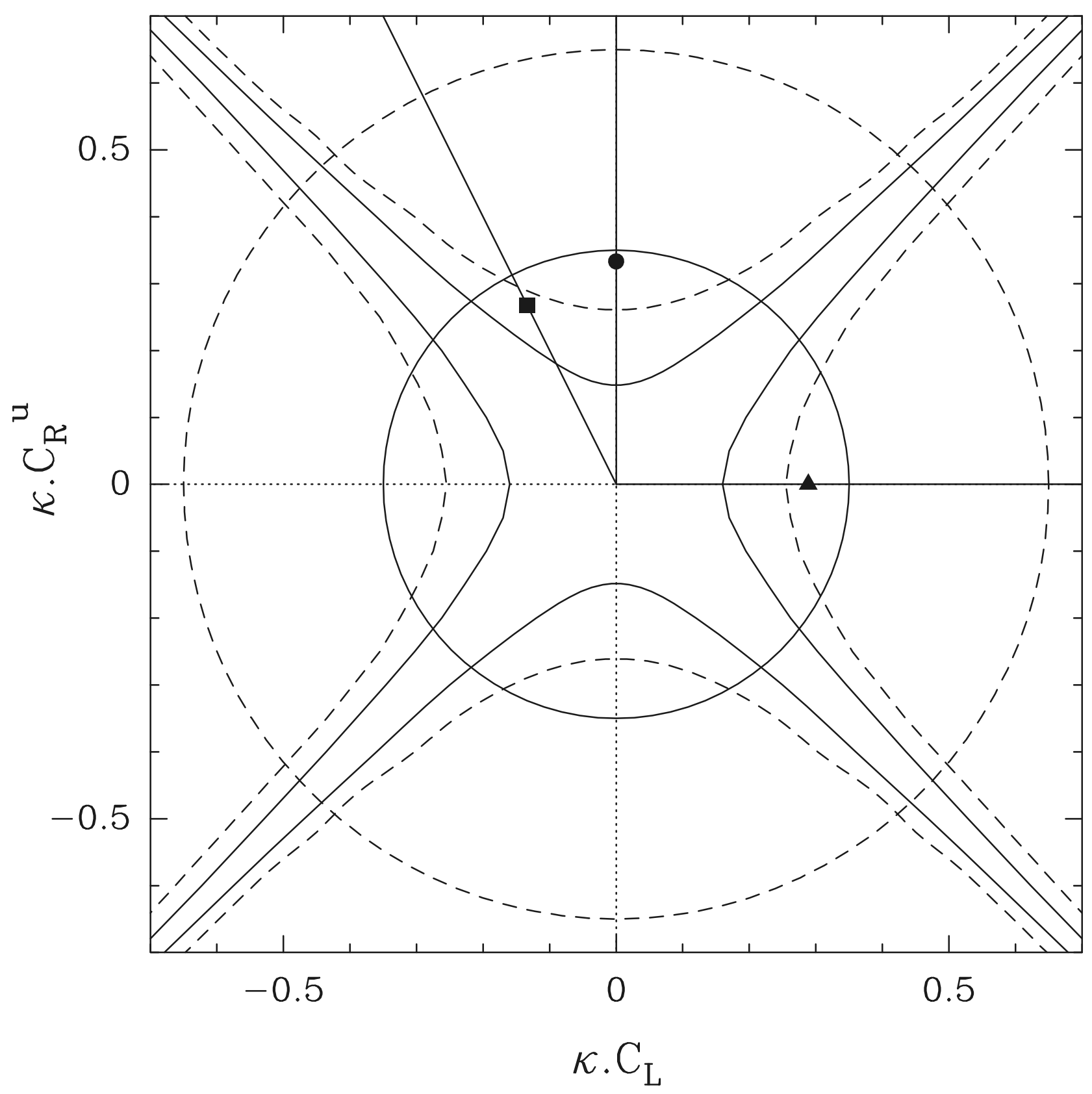

Fig 3 